\title{
Boccaccio e la Fantasia
}

\author{
Euisabetta MenetTi"
}

ABSTRACT: La fantasia nel Decameron di Giovanni Boccaccio che tra fabula e historia deve misurarsi immediatamente con le conseguenze della stretta relazione stabilita fin dall'antichità tra invenzione e verità, creando un testo in cui il mondo reale dialoga con il meraviglioso e il mirabile nella creazione di mondi altri in cui viaggia l'intera brigata dei dieci narratori. Si verifica anche come la letteratura per Boccaccio, che presenta la sua teoria nel Genealogia deorum gentilium, è locutio sub figmento capace di creare - per mirabile tensione creativa - un nuovo mondo di parole.

PAROLE CHIAVE: Giovanni Boccaccio; poesia; fantasia; storia; fabula; meraviglia.

RESUMO: A fantasia no Decameron de Giovanni Boccaccio entre fabula e historia, deve medir-se imediatamente com as consequências da estreita relação, estabelecida desde a antiguidade, entre invenção e verdade, dando origem a um texto em que o mundo real dialoga com o maravilhoso e o extraordinário na criação de outros mundos, pelos quais viaja o grupo dos dez narradores. Pode-se verificar ainda como a literatura para Boccaccio, que apresenta sua teoria sobre a escrita

\footnotetext{
${ }^{*}$ Università di Modena e Reggio Emilia
} 
em seu Genealogia deorum gentilium é locutio sub figmento capaz de dar vida-por admirável tensão criativa - a um mundo novo de palavras.

PALAVRAS-CHAVE: Giovanni Boccaccio; poesia; fantasia; história; fábula; maravilha.

ABSTRACT: In The Decameron, by Giovanni Boccaccio, fantasy between fable and history are immediately measured against the consequences of the close relationship, established since ancient times, between invention and truth. This producing a text in which the real world interacts with the wonderful and marvelous, in the creation of those other worlds through which the entire group of ten storytellers travel. It is also possible to verify, according to Boccaccio's theory presented in Genealogy Deorum Gentilium ("On the Genealogy of the Gods of the Gentiles"), that for him literature is locutio sub figment and it is able to conceive - by wonderful creative tension - a new world of words.

KEYWORDS: Giovanni Boccaccio; poetry; fantasy; history; fable; wonder. 


\section{La poesia è un fervore mirabile}

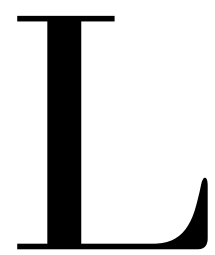

a parola fantasia ha nella sua radice l'idea dell'apparenza e della 'immagine, poiché deriva dalla radice indoeuropea ( $\mathrm{bha}$-) che si riferisce anche alla "luce" e a qualcosa che "brilla". Questa radice è riconducibile alla formazione di parole latine - come fama, fateor, for, fas, facundus e fabula - che sancisce la suggestiva e antichissima collaborazione creativa tra la lingua e la fantasia ${ }^{1}$.

Boccaccio era convinto che la mutevolezza della parola finzionale provenisse dal misterioso e indissolubile legame tra il linguaggio e la sua dimensione visionaria. In un suo testo teorico di grandissima importanza, il libro XIV della Genealogia deorum gentilium ${ }^{2}$, egli collega il termine

1 Queste considerazioni sono tratte dal saggio di G. Lepschy ancora attuale e ricco di suggestioni: Lepschy, G. Aspetti linguistici del fantastico, in Id. , Nuovi saggi di linguistica italiana, Bologna, il Mulino, 1989, pp. 199-230. Si veda anche: Corti, M. Percorsi dell'invenzione. Il linguaggio poetico e Dante, Torino, Einaudi, 1993, p. 6. Su questi argomenti rimando al mio: La realtà come invenzion. Forme e storie della novella italiana, Milano, Franco Angeli, 2015).

2 Le citazioni delle Genealogie si riferiscono da qui in avanti all'edizione curata da V. ZACCARIA: BOCCACCIO, G. Genealogie deorum gentilium, Voll. VII-VIII dell'opera completa diretta da BRANCA, V. (a cura di) Tutte le opere di Giovanni Boccaccio. Milano: Mondadori, 1998. Per le citazioni dal Decameron si veda: BOCCACCIO, G. Decameron, a cura di Vittore Branca, Torino, Einaudi 1992. 
confabulatio (conversazione) al termine fabula: "Fabula igitur ante alia a for faris honestam

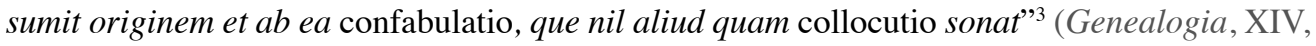
IX, 4)

Per Boccaccio, fabula è, prima di tutto, conversazione, è narrazione orale, è dialogo e scambio. E aggiunge: "Fabula est exemplaris seu demonstrativa sub figmento locutio, cuius amoto cortice, patet intentio fabulantis"4. È dote naturale delle donne e degli uomini parlare (locutio) e inventare (fabula): "Fabula est locutio". Siamo tutti, consapevolmente o meno, esseri narrativi, perché raccontare storie è insito nella natura umana.

Fabula è un modo di spiegare o rappresentare o di dimostrare con la parola qualcosa attraverso un processo di finzione (sub figmento). Nella lunga riflessione che compie nella Genealogia lo scrittore mette in campo alcune questioni teoriche dalla notevole tensione innovativa.

Per descrivere il mondo della fantasia Boccaccio si affida ad un termine (fabula) che declina in vario modo e che gli consente di mettere in luce gli aspetti cruciali del narrare nel rapporto tra veritas e fictio $^{5}$. Un rapporto che nasce tra l'intentio fabulantis (l'intenzione dell' autore o di chi parla attraverso le invenzioni) e le invenzioni stesse, metaforizzate nella "corteccia", che copre un'altra dimensione di significato.

Uno degli aspetti paradossali della lingua risiede curiosamente nella possibilità di una parola contenere in sé due significati razionalmente opposti. Un esempio della lingua italiana che fa al caso nostro è il chiaro caso di enantiosemìa della parola "storia", che viene definita nei vocabolari come "relazione vera" oppure "racconto inventato". Aspetto paradossale o "fantastico" del linguaggio e di una parola che dal racconto di Ulisse ai Feaci ai giorni nostri ha segnato e contrassegnato le riflessioni di scrittori, poeti, filosofi, storici, linguisti e in generale degli studiosi che si sono dedicati ai processi finzionali del racconto tra verità e menzogna.

Nel riflettere sulla poesia Boccaccio deve confrontarsi proprio con questa prima o primigenia ambiguità del racconto narrativo, di cui resta tuttora traccia nella parola italiana "storia". Per farlo egli sceglie un termine tecnico fabula che gli consente di fare un discorso generale sulla fantasia senza metterla in rapporto con il racconto storico e con i concetti di verità propri delle

3 La favola innanzitutto prende giusta origine da for-faris e da essa deriva confabulazione, che nient'altro significa ce colloquio.

4 La favola è un'espressione esemplificativa o dimostrativa sotto il velo della finzione; e rimossane la corteccia è manifesta l'intenzione di colui che fantastica

5 Fondamentale per una sistematica trattazione dei termini in questione: Segre, S. (Finzione, in Avviamento all'analisi del testo letterario, Torino, Einaudi,1985, p. 214-233, in particolare p. 214 e 215). 
categorie estetiche medievali ${ }^{6}$.

Fabula insomma gli consente di generalizzare sui processi finzionali di ogni tipo di racconto, mentre novella diventa il termine privilegiato per determinare un nuovo e particolare genere narrativo. Il primo nodo che deve sciogliere, dunque, riguarda il rapporto tra invenzione e verità, dove la veritas viene tradizionalmente intesa come una verità metafisica, trascendente o figurale.

Sulla base dei testi classici (Rethorica ad Herennium, De Inventione di Cicerone e Orazio) Boccaccio tenta di contrastare il giudizio più negativo che grava sul termine fabula: e cioè l'accusa di falsa apparenza o di falsità, che - se accolta - dimostrerebbe la pericolosità morale delle invenzioni dei poeti.

Inoltre egli deve affrontare i principi narrativi che governano il racconto esemplare della predicazione cristiana medievale secondo i quali l'invenzione, la fantasia, la favola sono usi strumentali di una narrazione che vuole trasmettere un messaggio pedagogico ed una verità.

In una prospettiva esoterica, infatti, solo i dotti riescono e possono penetrare il doppio strato del linguaggio figurale o, come scrive Boccaccio, la "corteccia" che copre "intentio fabulantis" ossia l'intenzione di chi fantastica.

Fabula, dunque, costringe Boccaccio a misurarsi immediatamente con le conseguenze della stretta relazione stabilita fin dall'antichità tra invenzione e verità e successivamente ripresa dalla patristica omiletica cristiana tra verità trascendentale e racconto esemplare.

Sono questioni che scottano perché investono direttamente la dignità del testo di invenzione. Un testo di invenzione o fabula il cui "effetto di realtà" o mimesi del reale è tradizionalmente vincolato al più ampio e complesso concetto teologico di veritas.

Ma Boccaccio - che non intende liquidare i racconti dei poeti come semplici "fantasticazioni" false e prive di verità - cerca una nuova strada. Alla classica tassonomia dei generi (fabula, historia e argumentum) propone di aggiungere una quarta tipologia, che prevede (e consente) il gioco totalmente illusorio dell'invenzione fantastica.

E tra fabula e historia egli intravede una gamma di possibili invenzioni che nascono dalla mescolanza delle due categorie estetiche, in base anche all'analisi delle invenzioni dei poeti classici latini e dei racconti delle Sacre Scritture. In questa sorta di paradigma del narrabile,

6 Si veda sulla "nebulosa" quanto ha chiaramente esposto C. Villa nella Discussione dal titolo Dante e il mondo classico: "La distinzione medievale tra fabula e historia non si riesce ad organizzare in un sistema coerente perché il Medioevo non tende a distinguere con la nettezza a noi consueta quello che è fabula da quanto è historia" (Seminario dantesco Internazionale International Dante Seminar 1. Atti del primo convegno tenutosi al Chauncey Conference Center, Princeton, 21-23 ottobre 1994, a c. di Z.G. Baranski, Firenze: Le Lettere, 1997, p.141). Fondamentale su questo argomento: MAZZACURATI, G. All'ombra di Dioneo. Tipologie e percorsi della novella da Boccaccio a Bandello, a cura di M. Palumbo, Firenze: La Nuova Italia, 1996. Sulle distinzioni terminologiche: SARTESCHI, S. Valenze lessicali di "novella", "favola", "istoria” nella cultura volgare fino a Boccaccio, in Favole, parabole, istorie. Le forme della scrittura novellistica dal Medioevo al Rinascimento. Atti del Convegno di Pisa 26-28 ottobre 1998, a cura di G.Albanese, L.Battaglia Ricci, R.Bessi, Roma: Salerno Editrice, 2000, pp. 85-108. Recentemente ritorna su questi punti, proseguendo la questione riaperta dal Mazzacurati: PALUMBO, M. Finzione e verità del racconto, in La letteratura e la storia. Atti del IX Congresso Nazionale dell'adi Bologna-Rimini 21-24 settembre 2005, a c. Di E.Menetti e C.Varotti, volume 1, Bologna, Archetipolibri2007, p. 207- 223. 
tratteggiato nella Genealogia tra classicità e cristianesimo, egli finisce per privilegiare la terza tipologia - più simile all'argumentum - che prevede una giusta ed equilibrata commistione di eventi, immagini, situazioni probabili.

Ma nel definire questa terza tipologia - quella che definisce dei "poeti famosi" - non vale la regola della veritas. Egli, così, si trova a scrivere chiaramente che le fictiones poetice (le finzioni poetiche) non sono menzogne. L'intenzione dei poeti non è di ingannare, ma solo di inventare.

Ecco i passaggi chiave per tutta la teoria del racconto occidentale delle origini: "Poetarum fictiones nulli adhereant specierum mendacii, eo quod non sit mentis eorum quenquam fingendo fallere; nec uti mendacium est, fictiones poetice, ut plurimum, non sunt nedum simillime, sed nec similes veritati" ; " Sic et poeta, quantumcunque fingendo mentiatur, mendacis ignominiam non incurrit, cum suum ufficium, non ut fallat, sed ut fingat iustissime exequatur"

I poeti, secondo Boccaccio, non sono obbligati al vincolo di verità. Non dicono menzogne ma inventano: il loro compito non è di ingannare. Esiste per Boccaccio una dimensione a parte, aerea, intangibile, mutevole della letteratura d'invenzione che non risiede nella realtà tangibile e che non è figura di una veritas trascendente.

La letteratura è locutio sub figmento capace di creare - per mirabile tensione creativa - un nuovo mondo di parole. I poeti hanno per Boccaccio una licenza speciale: la licentia vagandi.

I poeti, scrive, non "sono obbligati al vincolo di usare la verità nella superficie delle loro invenzioni e se venisse loro tolta la licenza di vagare per ogni genere di finzione il loro compito si risolverebbe proprio nel nulla"

Esiste infatti nei poeti la destrezza dell'artificium. I narratori non sono storici, ma ricostruiscono con "artificio molto più sottile" le dinamiche di una storia, di un avvenimento:

Nam poete non, ut hystoriographi faciunt, qui a quodam certo principio opus exordiuntur suum, et continua atque ordinata rerum gestarum descriptione in finem usque deducunt [...], verum artificio quodam longe maiori aut circa medium hystorie, aut aliquando fere circa finem inchoant quod intendunt, et sibi adinveniunt causam recitandi, quod ex procedentibus omisisse videbantur. ${ }^{9}$

Si apre così una nuova interpretazione dell'invenzione narrativa, libera dalle più rigide convenzioni dell'esemplarità e della veritas. Viene in queste pagine rivendicato in modo

7 Le invenzioni dei poeti non si accostano ad alcuna specie di mendacio, non avendo essi intenzione di ingannare poetando; né le finzioni poetiche sono somigliantissime, anzi neppure somigliano alla verità, come invece è il mendacio. 8 Così anche il poeta, per quanto finga nell'invenzione, non incorre nell'onta della menzogna, quando esegua onestamente il suo compito, che non è di ingannare [non ut fallat] ma di inventare [sed ut fingat].

9 I poeti infatti non fanno come gli storici, i quali da un certo principio cominciano la loro opera e con una continua e ordinata descrizione dei fatti la conducono fino alla fine ma con artificio molto più sottile, $\mathrm{o}$ circa a metà della storia, $\mathrm{O}$ talora quasi alla fine, incominciano a dire ciò che nelle parti precedenti sembravano aver omesso. 
stupefacente e modernissimo il diritto ad inventare, a fantasticare e a creare consapevolmente mondi altri che non per questo "tradiscono" il mondo reale.

La poesia è un "fervore mirabile" che permette ai poeti di catturare con poche lettere le forme, i costumi, i moti dei cieli, gli impetuosi fragori dei venti, il crepitio delle fiamme, i sonori mormorii delle onde e le ombre dei boschi. È un fervore che genera visioni mirabili attraverso l'allineamento, scrive, di poche lettere (parvis in licterulis).

\section{Realtà e finzione}

Il Decameron è lo specchio di questi ragionamenti teorici. Come è noto nell'Introduzione alla quarta giornata Boccaccio si difende polemicamente dalla fastidiosa accusa di falsità ${ }^{10}$. Il Decameron, scrive, è solo un mondo di parole:

Quegli che queste cose così non essere state dicono, avrei molto caro che essi recassero gli originali, li quali, se a quel che io scrivo discordanti fossero, giusta direi la loro riprensione e d'amendar me stesso m'ingegnerei; ma infino che altro che parole non apparisce, io gli lascerò con la loro oppinione, seguitando la mia, di loro dicendo quello che essi di me dicono. (Introduzione, IV, 39).

Fino a quando si tratterà solo di parole, la verità non può nemmeno essere verificata. Quindi, ogni finzione è legittima o forse ogni finzione contiene una particolare forma di verità. Gli aspetti della finzione, ricavati ora dalla tassonomia boccacciana (fabula, fictio, fabulantes, fictiones poetice) si incontrano con la fondazione della novella, un genere narrativo già sperimentato nell'anonimo Novellino duecentesco, che fa dell'aderenza alla realtà uno dei motivi fondanti della propria essenza e originalità.

Come è noto il cosiddetto "realismo" del Decameron - via interpretativa aperta dalla fondamentale lezione di Auerbach - è stato visto soprattutto nel grande affresco della vita quotidiana e in particolare nell'epopea dei mercanti (BRANCA), nella dimensione popolare e folclorica del linguaggio (BARBERI SQUAROTTI) e nella dimensione alta e ideale della brigata

$10 \mathrm{Si}$ vedano le fondamentali raccolte di Atti di Convegno: Malato (1989); Anselmi (1998); Albanese, Battaglia Ricci e Bessi (2000). Oltre alla bibliografia segnalata rimandiamo a questi studi più recenti: Bragantini (2012); Battaglia Ricci (2013). 
cortigiana (SURDICH) che è espressione di una nuova élite culturale (BATTAGLIA RICCI) ${ }^{11}$.

I tentativi di studiare gli aspetti più paradossali della lingua decameroniana sono stati intrapresi dai fondamentali studi di Muscetta sulla scia delle categorie di Michail Bachtin sul comico-grottesco, in parte successivamente ripresi dagli studi di Giancarlo Mazzacurati sulla novella italiana. Più recentemente Michelangelo Picone ha puntato sulla correlazione tra la cornice decameroniana e la cornice delle Mille e una Notte, mettendo in rilievo gli aspetti meno realistici e tutti orientali della dimensione narrativa molteplice e ricchissima di riferimenti incrociati e di fonti arabe.

Ma l'aspetto fantastico della narrativa medievale è stato soprattutto oggetto di studio degli storici del Medioevo e in particolare degli storici francesi: quelli di Jacques Le Goff, che su altri testi dell'epica francese ha delineato un percorso e una griglia tematica assai utile per rappresentare una mappa del fantastico e del meraviglioso medievali. Oltre a Le Goff anche Francis Dubost ha in modo completo illustrato molti degli aspetti dell'alterità fantastica nelle opere medievali narrative e in versi della letteratura francese. Gli studi teorici da quelli di Todorov a Zumthor, nonostante alcune divergenze metodologiche, hanno contribuito a chiarire le caratteristiche di fondo dello straniamento fantastico, attribuendo a questo evento o aspetto narrativo "fantastico" un carattere sovversivo o perturbatore del normale svolgimento dei fatti narrati.

Il Decameron è considerato dalla critica un testo dalla solida impostazione realistica, perché rappresenta e appunto esemplifica il mondo quotidiano della società medievale, gli usi e i costumi del popolo, ma anche gli usi e i costumi dei cavalieri, dei nobili, del clero o dei re.

Un grande affresco dell'umanità, si è detto, che restituisce in modo vivo e realistico la rappresentazione delle donne e degli uomini in un periodo storico preciso e ben individuato nella Peste Nera del 1348, esordio grandioso e terribile, dedicato alla morte, che avanza inesorabile.

Gli aspetti più aleatori della fantasia nel racconto novellistico nascono all'interno di questa rete immaginativa, in cui anche la rappresentazione realistica è parte fondamentale del gioco di invenzione. Già il De Sanctis, d'altronde, aveva chiaramente descritto l'atto rivoluzionario di questo scrittore nei confronti di quella che definisce la "letteratura teocratica" precedente, poiché la "cifra" del Decameron è il "maraviglioso, l'imprevisto, il fortuito, lo straordinario".

11 Si rimanda alla bibliografia essenziale. 
Boccaccio con la novella sperimenta, dunque, la compresenza delle due nature del linguaggio creativo e di invenzione: quello che permette di ricreare una realtà e quello che consente di alterare questa stessa realtà. Un gioco creativo con le parole: una nuova grammatica della fantasia e del narrare, che nel corso degli anni (dalla più tradizionale Amorosa Visione al Decameron al Corbaccio) verrà come abbiamo visto, teorizzata successivamente nel testo latino che abbiamo appena esaminato.

La fondazione di un nuovo concetto di fictio va di pari passo con la codificazione del nuovo genere narrativo, la novella, e quindi con la sperimentazione di una narrazione che contenga in sé contemporaneamente sia gli aspetti più prosaici o realistici d'ambiente sia situazioni narrative non realistiche, ma mirabili e inverosimili: aspetti insomma più propriamente meravigliosi o fantastici. È, dunque, con questa libertà dell'ingegno narrativo - coraggiosamente svincolato dal senso di verità - che lo scrittore dà vita alla rappresentazione di luoghi fantastici, piegando la lingua ad un nuovo immaginario e al linguaggio dell'alterità.

Mediante alcuni accorgimenti lessicali (l'invenzione di parole) e sintattici (la paratassi) o retorici (l'accumulazione) egli ricrea l'incantesimo di una lingua altra, proveniente da mondi lontani ed evocatrice di bellezze esotiche o da fiaba. Ma come avviene per l'affermazione della "nuova verità" dei poeti anche il "fantastico" viene colto su terreni diversi da quelli della tradizione.

A differenza, infatti, del fantastico medievale dei bestiari, dei mostri, dei diavoli, delle mirabili avventure cavalleresche o dei miracoli, il fantastico decameroniano si distingue per la distanza dalle caratteristiche formali del prodigioso soprannaturale cristiano e dalle simbologie allegoriche delle rappresentazioni iconografiche medievali fantastiche.

D'altronde all'interno di una schietta disposizione anticlericale, che mira a scardinare le astuzie dei predicatori e le falsità delle superstizioni religiose, il fantastico decameroniano doveva ovviamente ritagliarsi anche altri spazi.

Vediamo ad esempio la novella di Frate Cipolla (VI, 10): gli aspetti fantastici della sua celebre predicazione vengono recuperati sul piano della letterarietà e non su quello del soprannaturale cristiano, che viene invece ferocemente deriso. Quello di Frate Cipolla è un esercizio di abilità oratoria che sconfina nella dimensione surreale e straniata del linguaggio dell'alterità. È una predica o la parodia di una predica intessuta di allusioni a terre lontane ma anche a luoghi conosciuti e, tuttavia, straniati da un contesto assolutamente irreale. Qui il divertimento verbale è fondato sull'ironia dei caratteri del mondo rovesciato, che era il tratto caratteristico della 
letteratura di viaggio medievale.

Nel Decameron dunque la dimensione fantastica è nello stupore e nella meraviglia di chi ascolta e di chi legge. La novella è raccontata, narrata: è appunto confabulatio tra le giovani donne e i giovani della brigata. È una novitas che rappresenta la realtà, raccontandola sub figmento locutio.

L'evento mirabile è qualcosa che brilla: è una visione, un'immagine che proviene dal cuore delle donne e degli uomini. È soprattutto un fantasticare ad occhi aperti delle donne "malinconose" che cercano di guarire grazie ai loro sogni, alle loro visioni e alle loro immaginazioni.

La donna è al centro di questa sorta di liberazione fantastica: sono infatti le sette narratrici che riescono a coinvolgere i tre giovani narratori in una sorta di gara del fantasticare. E non è un caso che siano le narratrici ad inaugurare i grandi temi del fantastico:

- Pampinea inaugura il "fantastico avventuroso" con l'incredibile favola di Alessandro - è la II, 3 dove Alessandro, giovane spiantato, sposa dopo molte peripezie la figlia del re di Inghilterra;

- Filomena per prima sperimenta gli aspetti del "fantastico-macabro" con l'orribile descrizione del cadavere di Ambrogiuolo (II, 9);

- Di Filomena sono le novelle di Lisabetta da Messina (IV, 5), l'innamoratasognatrice, e di Nastagio degli Onesti $(\mathrm{V}, 8)$, protagonista della caccia infernale;

- Emilia è la prima ad accedere al magico: al giardino magico d'inverno di Dianora $(X, 5)$;

- Elissa si inventa la terra di Bengodi (VIII, 3), dove viene immaginata la terra dell'abbondanza.

Le donne sognano e inventano: a loro lo scrittore dedica il Decameron. Il luogo ideale in cui le donne narratrici risiedono per dar vita alle loro visioni è una valle meravigliosa: la valle delle donne. Una valle rigogliosa dove le bellezze naturali sono iperboliche e si sommano l'una all'altra in una vertiginosa accumulazione: vigne, ulivi, mandorli, ciliegi, fichi carichi di frutti 
e infiniti.

Ma ancora ombra di boschetti, di piccole querce, di frassini e di alberi verdissimi innumerevoli. Gli alberi formano un disegno ordinato come se un artista li avesse disposti in una sorta di affresco, e poi ancora una distesa di erba, minuta e fresca punteggiata da fiori di tutti i colori. Il sole è alto (è mezzogiorno) ma vi è l'ombra fresca di questo giardino delle meraviglie con cui ristorarsi.

Ed erano queste piagge, quante alla piaga del mezzogiorno ne riguardavano, tutte di vigne, d'ulivi, di mandorli, di ciriegi, di fichi e d'altre maniere assai d'alberi fruttiferi piene, senza spanna perdersene. Quelle le quali il carro di tramontana guardava, tutte eran boschetti di querciuoli, di frassini e d'altri alberi verdissimi e ritti quanto più esser poteano. Il piano appresso, senza aver più entrate che quella donde le donne venute v'erano, era pieno d'abeti, di cipressi, d'allori e d'alcuni pini sì ben composti e sì bene ordinati, come se qualunque è di ciò il migliore artefice gli avesse piantati; e fra essi poco sole o niente, allora che egli era alto, entrava infino al suolo, il quale era tutto un prato d'erba minutissima e piena di fiori porporini e d'altri. (Conclusione, VI, 1).

La valle delle donne è il paradiso in terra. Qui le giovani narratrici trovano un laghetto, si bagnano in acque limpidissime e i loro corpi, sensuali e liberi, si distendono e prendono vita. È una valle fiabesca, irreale, dove le sette narratrici, separate per un momento dai tre giovani, ritrovano se stesse durante la pausa narrativa tra la sesta e la settima giornata.

È il loro sogno di liberazione fantastica, di cui anche il loro corpo fa parte. Le donne rinascono così a nuova vita nella lotta terribile che stanno combattendo: la lotta dell'immaginazione fantastica, dell'invenzione contro la dura realtà di morte della Firenze appestata. La valle delle donne è quindi una visione colorata, irreale, luminosissima. Il locus amoenus, rifugio delle giovani sognatrici e dei giovani sognatori, è il luogo del narrare e del fantasticare. 


\section{La musica delle parole}

Nel Decameron il raccontare assomiglia a volte ad un cantare in quanto si cerca il piacere dell'udito. Agostino nel De musica collega il ritmo della misura delle sillabe a quello della musica, ipotizzando una scientia bene modulandi, che riguarda proprio la scansione ritmica della voce.

Il canto e la musica sono il primo rimedio della brigata contro il silenzio in cui è piombata la città di Firenze, dove chi muore di peste non ha "funeral pompa di cera e di canti" (Introduzione, I, 32). La nuova vita dei narratori, dopo il drammatico inizio di morte, viene inaugurata da un duetto musicale con Dioneo al liuto e Fiammetta alla viola.

Il primo sodalizio amoroso e creativo tra Dioneo/Boccaccio e la sua amata si celebra proprio con la musica e successivamente viene esteso agli altri componenti della brigata, che si uniscono a loro nella danza e nel canto. Inoltre prima ancora di raccontare i dieci giovani cercano un accordo comune con l'esercizio della musica, del canto e della danza, proprio come le Muse:

E levate le tavole, con ciò fosse cosa che tutte le donne carolar sapessero e similmente i giovani e parte di loro ottimamente e sonare e cantare, comandò la reina che gli strumenti venissero; e per comandamento di lei Dioneo preso un liuto e la Fiammetta una viuola, cominciarono soavemente una danza a sonare. Per che la reina coll'altre donne, insieme co' due giovani presa una carola, con lento passo, mandati i famigliari a mangiare, a carolar cominciarono; e quella finita, canzoni vaghette e liete cominciarono a cantare. (Introduzione, I, 105-107)

Opportunamente separati dalla servitù, gli eletti di Boccaccio sentono con il proprio corpo la musica, i suoni ed il ritmo, creando subito un'atmosfera festosa che si oppone al silenzio mortale che li circonda.

La musica e la danza, insomma, precedono l'atto narrativo fantastico come prima pratica ricreativa quotidiana della brigata, che ricorda una visione di ninfe danzanti. Qui, in effetti, Boccaccio fa economia e riusa uno stilema che aveva già messo alla prova nell'Amorosa Visione (A, canto XLI, v. 7-39) dove la musica, il carolare ed il lento passo diventano il tratto distintivo dell'incedere delle sue ninfe fiorentine che, così, si confondono con le giovani donne della brigata decameroniana. 
La fantasia di Boccaccio è una continua rielaborazione mitopoietica che combina immagini e situazioni dal mondo classico con quello contemporaneo. La sensualità delle ninfe, che si muovono sinuose con lento passo, serve a descrivere le attuali bellezze fiorentine, cioè le donne narratrici. Una musica straordinaria accompagna il ballo delle ninfe nell'Amorosa Visione così come introduce la lieta brigata nei racconti del Decameron.

Nell'Introduzione alla prima giornata la svolta dal silenzio alla musica segna il passaggio dalla morte alla vita. Dioneo e Fiammetta sono gli artisti che invitano il gruppo a superare il trauma della morte con l'arte delle muse. Dietro di loro, in trasparenza, si può intravedere la coppia-archetipo, formata dal musico Orfeo e dalla ninfa Euridice, qui riadattata a nuove finalità con la giocosa riconversione di Orfeo in Dioneo, che canta ed incanta la sua generazione.

Per Boccaccio il mito di Orfeo è il sommo esempio dell'incantesimo poetico ed è il punto di arrivo o di successo per ogni scrittore: nel sonetto Quel dolce canto delle Rime (XIX) il suono e la dolcezza sono legati insieme ad esprimere questa magica possibilità di incantamento.

Nel Decameron la musica non ha una sola dimensione ma viene evocata per esprimere situazioni molto diverse. Si trova la canzone folclorica ma anche la musica liturgica, usata soprattutto come occasione di rovesciamento comico-grottesco nelle novelle di beffe. Si incontra la musica della festa ma anche la semplice canzone che invita ad una riflessione profonda e malinconica in relazione anche all'Ars nova francese.

La versione comica e popolare della canzone è ricordata da Dioneo nella conclusione della quinta giornata grazie al suo divertito elenco di canzonacce popolari e grottesche. Persino il sempliciotto Calandrino è capace di suonare e di cantare con la sua "ribeba" canzoni allegre per tutta la compagnia (nella novella IX, 3). Non si intende ora ripercorrere tutte le occorrenze in cui la musica compare nel Decameron fin dalla prima giornata, dato che di recente sono state ampiamente documentate.

Si prenderanno in considerazione solamente alcuni significativi passi in cui Boccaccio sembra suggerire, in nome del mito di Orfeo, una riflessione sugli effetti che la musica produce sugli ascoltatori e sulla possibile correlazione con la pratica dell'ascolto della narrazione novellistica.

Una canzone può essere usata come un breve racconto che reca un messaggio d'amore: $\mathrm{i}$ 
messaggi galeotti sotto forma di canzonette - ricorda ironicamente Boccaccio in altri passi della Genealogia - sono all'ordine del giorno. Gli stessi nemici della poesia se le procurano da chi è capace di comporre in musica, visto che loro - ignoranti e insensibili e ipocriti (sic) - sono di povero ingegno. E, così, una canzone può commuovere, come avviene nella novella di Tedaldo degli Alisei e di monna Ermellina (III, 7), oppure può conservare negli anni il ricordo di un dramma amoroso, come avviene nella novella di Lisabetta da Messina (IV, 5). In questo ultimo caso, la novella si sviluppa da una canzonetta popolare ("Qual esso fu lo malo cristiano") che ha radici profonde nel sistema magico-religioso di rappresentazione del culto della sepoltura.

Nella decima giornata sono allineate, una dopo l'altra, due novelle (X, 6 e X, 7) che hanno al centro rispettivamente due re (re Carlo I d'Angiò e re Pietro d'Aragona), due frontiere culturali (la Napoli angioina e la Sicilia aragonese) e la musica.

La novella X, 6 è una visione ad occhi aperti del re Carlo d'Angiò che si perde in un giardino incantato. L'ambientazione è napoletana ma l'immaginario richiama immediatamente i romanzi cavallereschi d'Oltralpe: appaiono infatti due bellissime giovani, "Ginevra la bella" ed "Isotta la bionda", figlie di Neri degli Uberti, che incantano il vecchio re con una canzone eseguita con "tanta dolcezza e sì piacevolmente" che sembrano angeli scesi dal cielo.

L'incantamento del vecchio re aragonese assomiglia a quello del re angioino nel segno della dolcezza, dell'amore e della stupefazione della musica e del racconto. Non è, forse, difficile vedere in questa coincidenza di soggetto, di trama e di effetti anche un'affermazione di teoria poetica, soprattutto alla luce delle riflessioni già esposte della Genealogia. In queste due novelle vengono ribadite le coordinate ideologiche della nuova forma d'invenzione della novella che al fatto storico sovrappone un nuovo mondo e nuovi personaggi, del tutto irreali.

Le due narratrici, Fiammetta e Pampinea, tradiscono consapevolmente il vero storico e mettono in scena due re falsificati, quasi fiabeschi. A partire dalla realtà dei fatti storici, il genere novellistico, per sua natura ibrido ed ambivalente, attiva tutte le trame possibili e in esse la vera storia dei due re nemici, protagonisti dei Vespri siciliani, si offusca e si allontana.

Napoli e Palermo, le due città-simbolo dello scontro tra angioini e aragonesi, si trasformano in un luogo meraviglioso, quasi fiabesco, le cui immagini non provengono dalla ricostruzione storica degli ambienti ma al contrario da una creativa contaminazione dell'immaginario europeo e mediterraneo. Inoltre la fascinazione poetica e sensuale della decima giornata è accentuata dallo speciale sodalizio femminile: qui le donne della brigata sono definite per la prima volta "splendide" da Fiammetta la quale, non a caso, proprio sulla soglia della sua novella suggerisce 
alle ascoltatrici - escludendo per un istante la componente maschile - di abbandonare la noiosa disputa appena iniziata (su quale novella è da giudicare davvero magnanima) e di ascoltarla con una differente sensibilità femminile, più umile:

Splendide donne, io fui sempre in opinione che nelle brigate, come la nostra è, si dovesse sì largamente ragionare, che la troppa strettezza della intenzion delle cose dette non fosse altrui materia di disputare: il che molto più conviene nelle scuole tra gli studianti che tra noi, le quali appena alla rocca e al fuso bastiamo. $(\mathrm{X}, 6,3)$.

In queste parole si materializzano ancora una volta i due lettori del Decameron, il dotto umanista e la sedicente lettrice ingenua. Alleata di Dioneo, Fiammetta afferma chiaramente di non voler "disputare" di cose specialistiche ma di voler solo raccontare alle sue splendide donne ed a voce alta ("dire") le vicende di un re valoroso, il re Carlo.

Questo evidentissimo scarto tra storia e finzione non convince interamente le donne, dato che l'autore non manca di segnalare, nel passaggio tra una novella e l'altra, la polemica che serpeggia tra i giovani alla fine del primo racconto. La versione novellistica del magnanimo re Carlo d'Angiò, infatti, viene rifiutata da una 'anonima ghibellina' presente nella brigata: «quantunque alcuna, che quivi era ghibellina, commendar nol volesse» $(X, 72)$.

La novella successiva $(X, 7)$, raccontata da Pampinea, è dedicata all'avversario storico del re angioino - il re Pietro III d'Aragona, che compete con il nemico solo sul piano della morale - e è ambientata a Palermo, ma i personaggi che la animano sono fiorentini e toscani, a parte il re spagnolo. Questa novella contiene quel nobile intreccio tra malinconia, compassione e musica, che ci interessa in particolar modo.

La giovane Lisa è una donna malinconica, figlia di uno speziale di Firenze, che si è innamorata del re Pietro d'Aragona. Come le donne evocate nel Proemio, la giovinetta tiene le fiamme d'amore nascoste nel suo cuore e la malinconia la consuma. Lisa sa bene che il suo amore è impossibile ma, ad un certo punto, decide di confessare al re il suo segreto, sfruttando la disponibilità del padre ad accontentarla su ogni richiesta, pur di farla guarire dal male oscuro che la abbatte.

Avviene così che il padre invita a casa Minuccio d'Arezzo, noto musicista del tempo, che viene convinto dalla giovane a diventare il suo portavoce. Lisa, prima di attuare il suo piano segreto, gli chiede di poter ascoltare alcune canzoni: "e, poi che alquanto con amorevoli parole 
confortata l'ebbe, con una viola dolcemente sonò alcuna stampìta e cantò appresso alcuna canzone, le quali allo amor della giovane erano fuoco e fiamma là dove egli la credea consolare" $(\mathrm{X}, 7,11)$. La musica di Minuccio, però, non la consola affatto ma al contrario alimenta l'intensità del suo prepotente sentimento. Alla fine la donna gli chiede di comporre una canzone-messaggio che possa raccontare al re il suo tormento d'amore. Minuccio, con l'aiuto di un certo Mico da Siena, "buon dicitore in rima" compone la canzonetta "Muoviti amore e vattene a Messere" che è ad essere inserita per intero all'interno della diegesi novellistica. Inoltre essa è un breve racconto in versi di una sofferenza d'amore che, inserita nella novella in prosa, ha la funzione di un messaggio d'amore che comunica l'inconfessabile, cioè l'innamoramento di una suddita per un re.

Il musicista va a corte e canta, accompagnato dalla viola, questa canzone che suscita in tutti meraviglia e compassione:

Laonde egli cominciò sí dolcemente sonando a cantar questo suono, che quanti nella real sala n'erano parevano uomini adombrati, sí tutti erano taciti e sospesi a ascoltare, e il re poco piú che gli altri. $(\mathrm{X}, 7,25)$.

Alla fine, Minuccio rivela al re che la storia d'amore è attualissima ed è la trasformazione in suono della parola poetica: "non sono ancora tre giorni che le parole si fecero suono" $(\mathrm{X}, 7,25)$.. L'emozione profonda, indotta dalla musica, spinge il re alla compassione per un sentimento così intenso: "il re fece gran festa e commendò la giovane assa' e disse che di sì valorosa giovane si voleva aver compassione" (X, 7, 27). Il finale è da fiaba: il re, insieme con la regina, dona a Lisa la salute, la ricchezza (Cefalù e Caltabellotta) ed un marito. La canzone di Lisa ci rivela qualcosa di nuovo e di inaspettato.

Come è già stato notato dal primo commento di Vittore Branca, alcune parole del passo citato ricordano immediatamente il canto di Casella: "Amor che ne la mente mi ragiona'/cominciò elli allor sì dolcemente/ che la dolcezza ancor dentro mi suona" (Purgatorio, II, 112-114). Ma, come spesso accade, la citazione risveglia la memoria retroattiva dei lettori sui versi di poco precedenti, accendendo un nuovo collegamento tra la reazione di Dante e di Virgilio al canto di Casella e quella di Lisa, del re e della corte al canto di Minuccio. Il che ci obbliga a mettere ordine tra tutte queste emozioni letterarie.

Per Dante - riemerso in queste righe con un piccolo furto, "cominciò elli sì dolcemente" - la 
dolcezza della musica è consolazione - "che mi solea quetar tutte mie doglie" (Purgatorio, II, 108) -, mentre per la giovane è tormento - "allo amor della giovane erano fuoco e fiamma là dove egli la credea consolare" $(\mathrm{X}, 7,11)$. Oltre a questa ossimorica associazione bisogna segnalare anche la diretta filiazione che riguarda, invece, l'incantamento: Dante e Virgilio restano "tutti fissi e attenti" a sentire la musica di Casella, accumunati da una sorta di rapimento estatico della mente (Purgatorio, II, 115-119). Allo stesso modo i cortigiani ed il re della novella restano: "taciti, sospesi e adombrati".

La musica per Boccaccio sembra, in apparenza, la spontanea liberazione di un'emozione ed una naturale amplificazione del patire amoroso. Il controcanto dantesco, però, deve suggerire una certa cautela nel giudicare troppo modernamente questo suggestivo richiamo alle emozioni. L'incastonatura dell'ipotesto dantesco e la mistificazione del vero storico di due re - simbolo della dominazione francese e spagnola in Italia - sono due operazioni letterarie che hanno come primo scopo quello di agire sull'immaginario collettivo per gettare una nuova luce sulla finzione narrativa e sugli effetti dell'arte musicale e narrativa insieme. Questi elementi di rielaborazione testuale e storica sono determinanti per connotare letterariamente - e non psicologicamente - le novelle del Boccaccio.

Il canto non sempre consola, a volte tormenta e commuove, tuttavia questa umanissima riflessione dello scrittore non è solo un'ispirazione soggettiva ma si invera prima di tutto nel codice letterario di riferimento e punta ad illuminare un effetto speciale dell'arte e, cioè, lo straniamento. Uno straniamento emotivo che trova nell'esempio di Dante - ed ancora prima nel mito di Orfeo - la sua più autentica scaturigine.

In altre parole, nelle sue novelle Boccaccio (con i suoi alias, Mico da Siena e Minuccio d'Arezzo) desidera riattivare lo stesso effetto di stupefazione che trascina e addolcisce la nostra mente. A metà del Decameron Madonna Oretta (personaggio-protagonista della VI, 1) insegna al suo maldestro cavaliere che la novella è rispetto di un certo ritmo ed alla fine del libro, nell'atmosfera rarefatta della decima giornata, due narratrici della brigata aggiungono che l'esperienza artistica deve saper raggiungere l'effetto del rapimento e dello stupore.

Oretta, Fiammetta e Pampinea sono le donne-attrici che, seppure con funzioni interne al testo molto differenti, sono interpreti speciali di uno stesso spirito novellistico. Il loro esempio serve a Boccaccio per illuminare il prodigio di Orfeo: solo il narratore che conosce il ritmo musicale della prosa può dirsi amico delle Muse; e solo così il novellare potrà essere mirabilmente galeotto. 


\section{La fantasia, il meraviglioso e il mirabile}

Il richiamo al meraviglioso e al mirabile nella descrizione di luoghi naturali ideali e nella rappresentazione di situazioni ed eventi che alterano la norma producono nel lettore una sorta di straniamento. L'avventura - tema centrale della tradizione del romanzo cavalleresco d'oltralpe - dà vita al meraviglioso delle azioni e all'alterità mirabile: alla creazione cioè di mondi altri in cui viaggia l'intera brigata dei dieci narratori.

La seconda giornata in particolare, dedicata al tema della Fortuna, è ricca di narrazioni dal tono fiabesco: viaggi in terre lontane, peregrinazioni e spaesamenti, perdite e sorprendenti ritrovamenti, tesori dimenticati e poi recuperati, pirati ed eroine trascinati dai flutti.

Il Mediterraneo è una delle scenografie del fantastico boccacciano. Qui ad esempio possiamo ritrovare Landolfo Rufolo (II, 4), l'intrepido mercante che si trova a galleggiare alla deriva, aggrappato, senza saperlo, ad una cassa del tesoro.

Sempre in questo mare - teatro di avventure e di passioni - si perde e si ritrova la bellissima Alatiel (II, 7) che tra rapimenti, guerre, delitti e naufragi viene rapita e tenuta prigioniera per quattro anni da un castellano, da mercanti, da un principe, da un duca, dal figlio dell'imperatore di Costantinopoli, dal re dei Turchi, dal suo "famigliare" fino all'incontro risolutivo con un suddito del padre, che la riporta a casa.

Lo stesso nome Alatiel (o "la lieta") è costruito come l'eco fantastico di un nome arabo; così come il padre di Alatiel: Beminedab.

Beminedab e Alatiel provengono dalle terre d'oriente che sono promesse d'avventura, di ricchezza e di bellezza. E Alatiel - primo modello di giovane donna in cerca di avventure e di amore sensuale - usa il proprio corpo al posto della parola, proprio perché non conosce i linguaggi "stranieri” con cui viene in contatto durante le sue peregrinazioni mediterranee.

Da Alessandria naufraga nell'isola di Maiorca, poi viene trascinata in Grecia e si muove tra le Sporadi, Smirne, Rodi e infine Cipro. In questa avventura stupefacente la "vergine" Alatiel conosce l'amore, anzi conosce più amori, di re e imperatori. È una donna spaesata, che attraverso l'alterità e la sessualità ritrova felicemente se stessa. Viaggiare con Alatiel è come un sogno che si realizza per le "donne malinconose", lettrici ideali alle quali si rivolge il nostro scrittore.

Altro tema fantastico è l'incantesimo. Il viaggio da oriente e occidente è realisticamente molto lungo per un uomo del Medioevo. Ed ecco che messer Torello viaggia in una notte dalla corte del Saladino a casa sua, a Pavia. 
Il mezzo del viaggio fantastico è un letto magico. Il racconto dello straordinario incantesimo che permette a messer Torello, prigioniero del Saladino, di tornare a casa sua si trova nella decima giornata dedicata in modo significativo alla magnificenza e alla magnanimità. La novella si trova a poca distanza da un altro episodio di magia, quello del giardino fiorito a gennaio di Madonna Dianora.

Torello vola da Oriente a Occidente immerso in un sonno profondo. Dopo aver bevuto la pozione magica del negromante, scompare dalla vista del Saladino e ricompare, adagiato su un letto e colmo di ricchezze di ogni genere e vestito alla saracina, con un turbante in testa, nella chiesa della sua città, Pavia, immersa nell'ombra e nel silenzio: lo sfolgorare del letto orientale è l'evento fantastico che sorprende e spaventa. I frati fuggono di fronte all'evento incomprensibile e all'apparizione straordinaria di un personaggio delle Mille e una Notte.

Il tesoro di Torello è inestimabile ed è elencato con dovizia di particolari dal narratore. L'enumerazione meravigliosa si sofferma sugli oggetti che si accumulano sul letto e sulla pagina: corona, anelli, spade, pietre preziose, fermagli, coppe piene di oro. L'abbondanza e la ricchezza sono rese dall'enumerazione paratattica di tutti i gioielli che vengono nominati con un crescente tono iperbolico.

Appresso mise in dito a messer Torello uno anello, nel quale era legato un carbunculo, tanto lucente che un torchio acceso pareva, il valor del quale appena si poteva stimare; quindi gli fece una spada cignere, il cui guernimento non si saria di leggieri apprezzato; e oltre a questo un fermaglio gli fé davanti appiccare, nel qual erano perle mai simili non vedute, con altre care pietre assai; e poi da ciascun de' lati di lui due grandissimi bacin d'oro pieni di dobre fé porre, e molte reti di perle e anella e cinture e altre cose, le quali lungo sarebbe a raccontare, gli fece metter da torno. E questo fatto, da capo baciò messer Torello, e al nigromante disse che si spedisse; per che incontanente in presenzia del Saladino il letto con tutto messer Torello fu tolto via, e il Saladino co' suoi baroni di lui ragionando si rimase..

Infine, un ultimo tema importante del fantastico nel Decameron: il sogno, la visione. Il narrare fantastico è visione ed è luce: due elementi che si trovano nelle immagini oniriche. Boccaccio ancora una volta affronta nel Decameron il tema della visione in modo differente 
dalla tradizione scolastica medievale, ricchissima, come è noto, di visioni allegoriche.

Ma per lo scrittore che inventa - e non scrive menzogne -, non è il linguaggio allegorico della visione ad attrarre la sua attenzione, ma il sogno segreto che si nasconde nel cuore delle donne e degli uomini.

La dimensione notturna dell'uomo, svincolata dal dialogo ultraterreno, è rappresentata pienamente nel Decameron (ma anche nelle altre opere) come flusso creativo, mirabile, "impossibile" e "vano" del mondo interiore. È il sogno di Lisabetta da Messina, di Talano d'Imole, di Andreuola e di Gabriotto. Ma è anche la visione infernale di Nastagio degli Onesti, che è un'apparizione fantastica e rappresentazione teatrale della sofferenza e del delitto d'amore.

I sogni, che rappresentano soprattutto le nostre angosce, si possono avverare. È il caso nel disperato sogno di Lisabetta da Messina, che ritrova in sogno il cadavere dell'amato, ucciso dai fratelli. Lisabetta non sa che Lorenzo è morto, ma - come accade nei lutti - lo "sente": e lo sogna, vivo ma diverso: pallido, rabbuffato, con i panni stracciati che testimoniano la violenza subita.

Avvenne una notte che, avendo costei molto pianto Lorenzo che non tornava, ed essendosi alla fine piagnendo addormentata, Lorenzo l'apparve nel sonno, pallido e tutto rabbuffato e con panni tutti stracciati e fracidi indosso, e parvele che egli dicesse:

- O Lisabetta, tu non mi fai altro che chiamare e della mia lunga dimora t'attristi, e me con le tue lagrime fieramente accusi; e per ciò sappi che io non posso più ritornarci, per ciò che l'ultimo dì che tu mi vedesti i tuoi fratelli m'uccisono. E disegnatole il luogo dove sotterrato l'aveano, le disse che più nol chiamasse né l'aspettasse, e disparve. La giovane destatasi, e dando fede alla visione, amaramente pianse. (IV, 5 ,

La visione, rappresentata dai richiami ai verbi apparire e sembrare, è veritiera. Lisabetta ritrovasi sveglia, dà fede alla visione, trova il cadavere e, come si sa, tenterà di ridare vita, sotto le sembianze di una profumata pianta di basilico, all'amato scomparso. Anche questa una delicata e mirabile immagine di metamorfosi di ovidiana memoria.

Raccontare è immaginare e condividere. È rappresentazione visionaria di un mondo altro, che non deve essere giudicato con i tradizionali concetti di veritas teologica. Il fantastico proviene 
dai sogni e dalle apparizioni fantasiose di avventure, di mirabili avvenimenti, di capovolgimenti di fortuna, di liberazioni e di speranze. Sono novelle "artificiose" - cioè fantastiche -, pensate da narratrici e da narratori che usano pienamente e felicemente la licentia vagandi.

Queste novelle antiche possono mostrare ancora oggi, dopo tanti secoli, che la fantasia è la vera forza che coinvolge e trascina chi legge. Per noi, lettori del duemila, la lezione del Boccaccio è che l'incantesimo di ogni racconto risiede nella capacità dei poeti di far risuonare la musica delle loro parole per chi è capace di ascoltarle. Se esiste una remota possibilità di salvezza dalla morte, ci dice Boccaccio, essa risiede nella virtù più potente che ha a disposizione l'umanità per annullare il peso del male, della morte e dell'oblio: una forma di saggezza fantastica che è il sapere immaginare, il sapere raccontare ed il sapere ascoltare.

\section{Bibliografia ragionata}

Le citazioni dei testi di Boccaccio hanno come riferimento le edizioni delle opere dell'autore curate da Vittore Branca.

BRANCA, V. ( a cura di) Tutte le opere di Giovanni Boccaccio. Milano: Mondadori, 1998.

BOCCACCIO, G. Decameron, a cura di Vittore Branca, Torino, Einaudi 1992.

Tra i saggi riguardanti il Decameron e la novellistica si vedano in particolare i testi classici:

AUERBACH, E. Frate Alberto. In Mimesis. Il realismo nella letteratura occidentale (1946).Torino: Einaudi, 1956.

BARATTO, M. Realtà e stile nel "Decameron". Vicenza: Neri Pozza, 1970.

BARBERI SQUAROTTI, G. Il potere della parola. Studi sul "Decameron”. Napoli: Federico e Ardia, 1983.

BATTAGLIA RICCI, L. Ragionare nel giardino. Boccaccio e i cicli pittorici del "Trionfo della Morte". Roma: Salerno, 1987.

BRAGANTINI, R.; FORNI, P. M. (a cura di). Lessico critico decameroniano. Torino: Bollati Boringhieri, 1995.

BRANCA, V. Boccaccio medievale e nuovi studi sul Decameron (1970). Firenze: Sansoni, 1990.

BRUNO PAGNAMENTA, R. L'ambiguità come strategia narrativa. Ravenna: Longo, 1999.

CAPPELLO, G. La dimensione macrotestuale. Dante, Boccaccio, Petrarca. Ravenna: Longo, 1988. 
CARDINI, F. Le cento novelle contro la morte. Giovanni Boccaccio e la rifondazione cavalleresca del mondo. Roma: Salerno, 2007.

CHERCHI, P. L'onestade e l'onesto raccontare del «Decameron». Fiesole: Cadmo, 2004.

CURSI, M. Il Decameron: scritture, scriventi, lettori. Storia di un testo. Roma: Viella, 2007.

GETTO, G. Vita di forme e forme di vita nel Decameron. Torino: Petrini, 1958.

GUGLIELMINETTI, M. Boccaccio e la novella italiana. In Il tesoro della novella italiana. Milano: Mondadori, 1986.

. La cornice e il furto. Studi sulla novella del '500. Bologna: Zanichelli, 1984.

Sulla novella italiana. Genesi e generi. Lecce: Milella, 1990.

LAVAGETTO, M. (a cura di). Il testo moltiplicato. Lettura di una novella del «Decameron». Parma: Pratiche, 1983.

MENETTI, E. Boccaccio e la fictio. Studi sul Boccaccio, v. XXXVIII, p. 69-87, 2010. .Il "Decameron" fantastico. Bologna: Clueb, 1994. . La fucina delle finzioni. Le novelle e le origini del romanzo. Heliotropia, v. 8-9, p. 17-34, 2012.

PADOAN, G. Il Boccaccio, le Muse, il Parnaso e l'Arno. Firenze: Olschki, 1978.

PICONE, M. (a cura di). Autori e lettori di Boccaccio. In Atti del Convegno Internazionale di Certaldo, 20-22 settembre 2001. Firenze: Franco Cesati, 2002.

Boccaccio e la codificazione della novella. Letture del «Decameron». Ravenna: Longo, 2008. . Il racconto. In BRIOSCH,I F.; DI GIROLAMO, C. (a cura di). Manuale di letteratura italiana. Storia per generi e problemi. v. I. Torino: Bollati Boringhieri, 1993.

PICONE, M.; MESIRCA, M. (a cura di). Introduzione al Decameron. Firenze: Franco Cesati, 2004. PORCELLI, B. Dante maggiore e Boccaccio minore. Strutture e modelli. Pisa: Giardini, 1987.

SANGUINETI, E. Gli "schemata" del "Decameron". In RISSO, E. (a cura di). Il chierico organico. Scritture e intellettuali. Milano: Feltrinelli, 2000.

SEGRE, C. Funzioni, opposizioni e simmetrie nella giornata VII del "Decameron". In Le strutture e il tempo. Torino: Einaudi, 1974.

STORINI, M. C. Lo spazio dell'avventura. Peripezia e racconto nel Medioevo. Firenze: La Nuova Italia, 1997.

SURDICH, L. La cornice d'amore. Studi sul Boccaccio, Pisa, ETS, 1987.

VEGLIA, M. “La vita lieta”. Una lettura del "Decameron”. Ravenna: Longo, 2000. 
Nelle maggiori storie letterarie si possono trovare studi complessivi sull'opera di Giovanni Boccaccio che sono utili per un inquadramento generale. Con alcune parti antologiche commentate si veda di C. Muscetta (1972) e la voce di V. Branca: Boccaccio Giovanni per il Dizionario critico della letteratura italiana (1986):

BRANCA, V. (a cura di). Dizionario critico della letteratura italiana. v. I. Torino: UTET, 1986, p. 345-361.

MUSCETTA, C. Boccaccio. In La Letteratura Italiana. Storia e testi. Bari: Laterza, 1972.

Nella Letteratura Italiana Einaudi si possono leggere due saggi, il primo comparativo fra le 'tre corone' di R. Mercuri (1987) e il secondo di Asor Rosa (1992) finalizzato al capolavoro novellistico:

ASOR ROSA, A. Decameron di Giovanni Boccaccio. In Letteratura italiana, Le opere. v. I. Torino: Einaudi, 1992, p. 473-591.

MERCURI, R. Genesi della tradizione letteraria italiana in Dante, Petrarca e Boccaccio. In Letteratura italiana. Storia e Geografia. v. I. Roma-Bari: Laterza, 1987, p. 229-455.

Di pochi anni successivo il saggio sul «Decameron» di M. Picone (1995):

PICONE, M. Decameron. In BRIOSCHI, F.; DI GIROLAMO, C. (a cura di). Manuale di letteratura italiana. Storia per generi e problemi. v. I. Torino: Bollati Boringhieri, 1995, p. 625-654.

Dagli anni 1990 al 2000 si sono intensificati i saggi complessivi sulle opere di Boccaccio con diverse aperture interpretative. Fra queste si segnala il saggio di F. Bruni (1990), dedicato anche alla formazione di una nuova idea di letteratura, la letteratura 'mezzana', che si va definendo, in modo anche contraddittorio, tra le diverse opere

BRUNI, F. Boccaccio. L'invenzione della letteratura mezzana. Bologna: il Mulino, 1990.

Alla fine degli anni 1990 si segnala i volumi di:

BATTAGLIA RICCI, L. Boccaccio. Roma: Salerno, 2000.

SURDICH, L. Boccaccio. Roma; Bari: Laterza, 2001.

TATEO, F. Boccaccio. Roma; Bari: Laterza, 1998.

Gli studi complessivi sull'autore si intrecciano con gli studi sul genere novellistico delle origini che sono stati raccolti in alcuni importanti studi, che sconfinano nella letteratura novellistica del Rinascimento e del tardo Rinascimento. Una svolta negli studi della novellistica, per un nuovo approccio sulla formanovella e sulla sua evoluzione, si deve a G. Mazzacurati (1996).

MAZZACURATI, G. All'ombra di Dioneo. Tipologie e percorsi della novella da Boccaccio a Bandello. A cura di M. Palumbo. Firenze: La Nuova Italia, 1996.

Tra le raccolte di atti di Convegno si segnalano: 
ALBANESE, G.; BATTAGLIA RICCI, L.; BESSI, R. (a cura di). Favole, parabole, istorie. Le forme della scrittura novellistica dal Medioevo al Rinascimento. In Atti del Convegno di Pisa, 26-28 ottobre 1998. Roma: Salerno, 2000.

ALFANO, G. Nelle maglie della voce. Oralità e testualità da Boccaccio a Basile. Napoli: Liguori, 2006.

ANSELMI, G. M. (a cura di). Dal primato allo scacco. I modelli narrativi italiani tra Trecento e Seicento. Roma: Carocci, 1998.

CARAPEZZA, S. Novelle e novellieri. Forme della narrazione breve nel Cinquecento. Milano: LED, 2011.

MAURIELLO, A. Dalla novella "spicciolata" al "romanzo". I percorsi della novellistica fiorentina nel secolo XVI. Napoli: Liguori, 2001.

Si segnalano, inoltre, i seguenti saggi:

ALFANO, G. Comico in progresso: la funzione poietica della cornice decameroniana. In Nuova rivista di letteratura italiana, III, 2000, p. 99-119.

BRAGANTINI, R. Ingressi laterali al Trecento maggiore. Dante, Petrarca, Boccaccio. Napoli: Liguori, 2012.

CURTI, E. L"“Elegia di Madonna Fiammetta” e gli "Asolani” di Pietro Bembo. Alcune osservazioni sulle postille bembesche al codice Ambrosiano D 29 inf. Studi sul Boccaccio, XXX, p. 247-297, 2002.

KIRKHAM, V. Fabulous Vernacular. Boccaccio's "Filocolo" and the Art of Medieval Fiction. Ann Arbor: The University of Michigan Press, 2001.

MENETTI, E. Il Decameron tra divagazione e conoscenza. In BENISCELLI, A.; MARINI, Q.; SURDICH, L. (a cura di). La Letteratura degli Italiani. Rotte, confini, passaggi. Novi Ligure: Città del Silenzio, 2012, p. 29-46.

PALUMBO, M. Finzione e verità del racconto. In MENETTI, E.; VAROTTI, C. (a cura d). La letteratura e la storia. Atti del IX Congresso Nazionale dell'ADI Bologna-Rimini 21-24 settembre 2005, Bologna: Gedit, 2007, p. 207-223.

SURDICH, L. Il patto romanzesco nel Boccaccio. In COSTA, S.; DONDERO, M.; MELOSI, L. (a cura di). Le forme del narrare. Atti del VII Congresso Nazionale dell'Adi Macerata, 24-27 settembre 2003, Firenze: Edizioni Polistampa, 2004.

Per il dibattito intorno alle categorie fantastiche medievali:

BALTRUSAITIS, J. Il Medioevo fantastico. Milano: Adelphi, 1993. Risvegli e prodigi. La metamorfosi del gotico. Milano: Adelphi, 1999.

LE GOFF, J. Il meraviglioso e il quotidiano nell'occidente medievale. Bari: Laterza, 1990. 
ZUMTHOR, P. Semiologia e poetica medievale. Milano: Feltrinelli, 1973.

Su questi temi si veda:

MAZZOCUT-MIS, M. (a cura di). Deformazioni fantastiche: introduzione all'estetica di Jurgis Baltrušaitis: con due saggi dell'autore e una bibliografia completa delle sue opere. Milano: Mimesis, 1999.

Per l'impostazione sul tema della fantasia:

CALVINO, I. Lezioni americane. Sei proposte per il prossimo millennio. Milano: Garzanti, 1988.

CORTI, M. Percorsi dell'invenzione. Il linguaggio poetico e Dante. Torino: Einaudi, 1993.

LEPSCHY, G. Aspetti linguistici del fantastico. In . Nuovi saggi di linguistica italiana. Bologna: il Mulino, 1989, p. 199-230. 